\title{
The use of visual approach in teaching and learning the epsilon- delta definition of continuity
}

\author{
Pešić Duška ${ }^{1}$ and Pešić Aleksandar2,* \\ ${ }^{1}$ Information Technology School, Belgrade, Serbia \\ 2,* Faculty for Business and Industrial Management, Union University, Belgrade, Serbia \\ For correspondence: duskapesic@its.edu.rs
}

\begin{abstract}
In this paper we introduce a new collaborative technique in teaching and learning the epsilon-delta definition of a continuous function at the point from its domain, which connects mathematical logic, combinatorics and calculus. This collaborative approach provides an opportunity for mathematical high school students to engage in mathematical inquiry outside of the classroom by completing exercises that are based on the idea of visualization. Thus, the proposed approach enables cultivation of mathematical creative thinking and helps students to build their mathematical knowledge about the concept of a continuous function.
\end{abstract}

Keywords: continuous function, visualization, mathematical logic, high school students

\section{Introduction}

Our experience shows that many students in mathematical high schools have difficulties to understand the notion of continuity of the function at the point from its domain, which we believe may be due to the abstractness of the concept or the formal way of concept presentation. To try to deal with that problem we re-examine our teaching methods and emphasize the role of multifaceted approach in the teaching of our students, particularly in the teaching and learning of the epsilon-delta definition of a continuous function.

In this paper, we try to cover this particular concept in greater depth and also to create collaborative environments that encourage and support knowledge construction by students. In that sense, proposed approach has a twofold purpose: building new mathematical knowledge and developing student creativity by advancing their mathematical reasoning.

Generally, collaborative working with students implies that the teacher does not only act as facilitator in the teaching process but also establish partnership in working with students. When the teaching process promotes partnership, critical thinking and when it is consistent with constructivism, then working alone or in the groups, outside of the classroom and making mistakes could be considered as part of the learning process. Thereby, students could be encouraged to develop new knowledge and to take responsibility for their learning.

Our suggestion is that introduction of the concept of continuity should be included in the curriculum by relying on intuitive notion that students have about it (with particular emphasis on the issues that might appear to be in conflict with that notion). (Tall et al., 1981)

\section{Visual presentation}

Based on the assumption that appropriate visual explanations might be of great help in building an accurate and complete understanding of the concept of continuity, we proposed a visual presentation 
in our teaching-learning process.

After introducing the notion of continuity to the students (Takači et al., 2006), we argued that it is necessary to analyze in detail the epsilon delta definition of a continuous function at the point from its domain and systematically clarify all parts of that definition.

Definition 1. A function $f: D_{f} \rightarrow R, D_{f} \subset R$ is continuous at a point $x_{0} \in D_{f}$ if

$$
(\forall \varepsilon>0)(\exists \delta>0)\left(\forall x \in D_{f}\right)\left(\left|x-x_{0}\right|<\delta \Rightarrow\left|f(x)-f\left(x_{0}\right)\right|<\varepsilon\right)
$$

Students face difficulties making sense of the following notations (Takači at al., 2003):

- symbols $(\varepsilon$ and $\delta)$;

- quantifiers $(\forall$ and $\exists$ );

- inequalities $\left(\left|x-x_{0}\right|<\delta\right.$ and $\left.\left|f(x)-f\left(x_{0}\right)\right|<\varepsilon\right)$.

In order to overcome this problem, we propose the following visual explanations.

Let us suppose that $y=f(x)$ is a given function and $x_{0}$ is considered point from its domain. Then: $2 \delta, \delta>0$ is the width of the part of the $x y$-plane which lies between two vertical lines: $x=x_{0}-\delta$ and $x=x_{0}+\delta$, surrounding the line $x=x_{0}$ (denoted with V) (Figure 1).

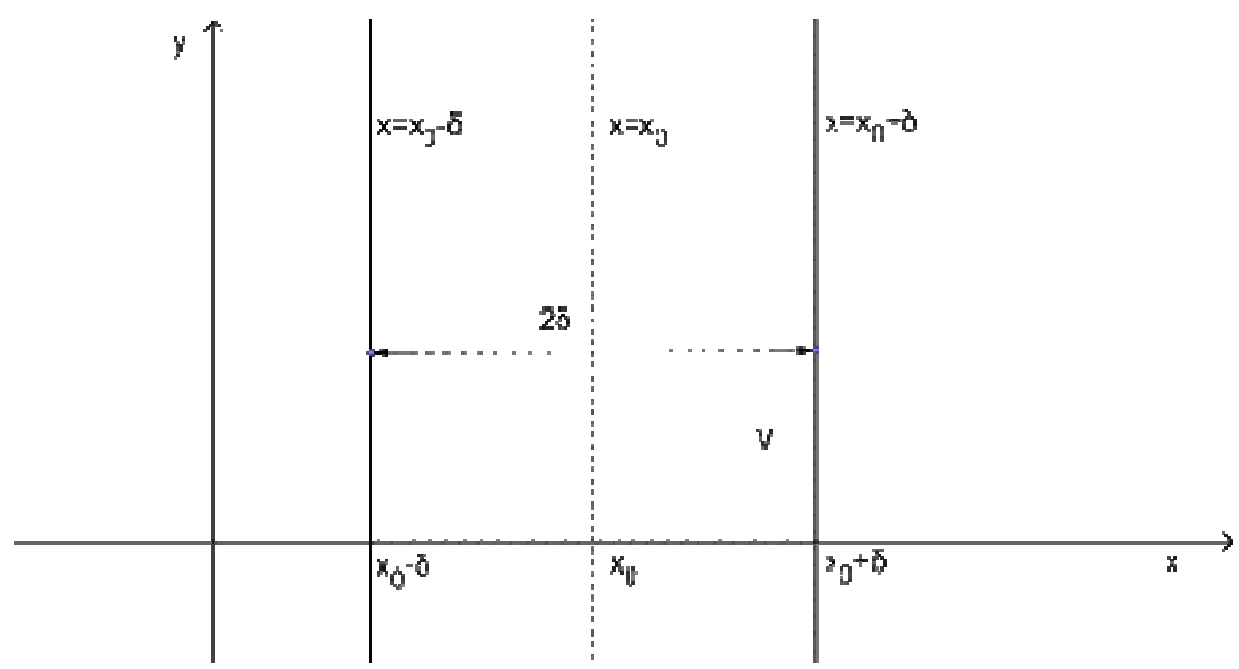

Figure 1. Visual presentation of the solution of the inequality $\left|x-x_{0}\right|<\delta$

$2 \varepsilon, \varepsilon>0$ is the height of the part of the xy-plane which lies between two horizontal lines: $y=f\left(x_{0}\right)-\varepsilon$ and $y=f\left(x_{0}\right)+\varepsilon$, surrounding the line $y=f\left(x_{0}\right)$ (denoted with H) (Figure 2); 


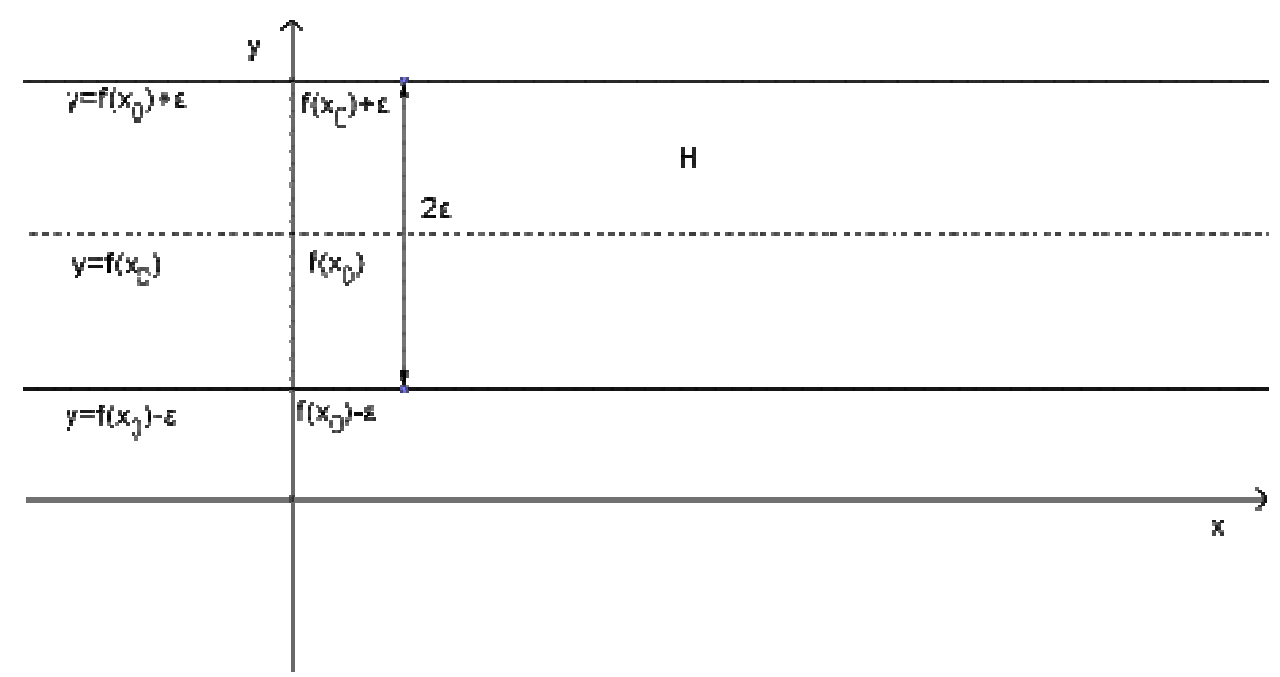

Figure 2. Visual presentation of the solution of the inequality $\left|f(x)-f\left(x_{0}\right)\right|<\varepsilon$

In that case, the visual explanations of the inequalities from the Definition 1 could be:

When the solutions of the inequality $\left|x-x_{0}\right|<\delta$ stands (i.e. $x_{0}$ belong to the interval $\left.\left(x_{0}-\delta, x_{0}+\delta\right)\right)$ then the corresponding set of points $(x, f(x))$ (the points of the Figure of the function) belongs to the area V (Figure 1).

When the solutions of the inequality $\left|f(x)-f\left(x_{0}\right)\right|<\varepsilon$ stands (i.e. $f\left(x_{0}\right)$ - belong to the interval $\left.\left(f\left(x_{0}\right)-\varepsilon, f\left(x_{0}\right)+\varepsilon\right)\right)$ then the corresponding set of points $(x, f(x))$ (the points of the Figure of the function) belongs to the area $\mathrm{H}$ (Figure 2).

The condition $\forall x \in D_{f}$ is very important, because if we replace $\forall x \in D_{f}$ by, say, $\exists x \in D_{f}$ in this definition, or if we completely leave this condition out, the new definition cannot assure the continuity of the function at the point.

For example, the function:

$$
f(x)=\left\{\begin{array}{cc}
\sin \left(\frac{1}{x}\right), & x \neq 0 \\
0, & x=0
\end{array}\right.
$$

satisfies all conditions of the following two formulae:

- $(\forall \varepsilon>0)(\exists \delta>0)\left(\left|x-x_{0}\right|<\delta \Rightarrow\left|f(x)-f\left(x_{0}\right)\right|<\varepsilon\right)$,

- $(\forall \varepsilon>0)(\exists \delta>0)\left(\exists x \in D_{f}\right)\left(\left|x-x_{0}\right|<\delta \Rightarrow\left|f(x)-f\left(x_{0}\right)\right|<\varepsilon\right)$,

but function $f(x)$ does have a discontinuity at the point $x_{0}=0$.

Obviously, only the precise order of all mentioned elements in Definition 1 ensures the continuity of the function at a point from its domain. In order to explain this, and to make it more clear, it is convenient to analyze in details all formulae resulting from the epsilon-delta definition by changing the order of these elements.

First of all, it is important to mention the following two formulae:

1) $(\forall \varepsilon>0)(\exists \delta>0)(P)$,

2) $(\exists \delta>0)(\forall \varepsilon>0)(P)$.

The quantifiers in these formulae are given in a different order.

In formula $1, \delta$ depends on $\varepsilon$, while in formula $2, \delta$ does not depend on $\varepsilon$. Therefore, these two formulae have completely different meanings.

As example, the following two formulae are suitable to be compared: 
3) $(\forall n \in N)(\exists m \in N)(m \geq n)$,

4) $(\exists m \in N)(\forall n \in N)(m \geq n)$.

The meaning of formula 3 is: 'There is no biggest natural number'.

If we change the order of elements, we get the formula 4 . This formula has completely opposite meaning: 'There is the biggest natural number'.

It is also important to clear up the meaning of implication $p \Rightarrow q$, and to emphasize that it is correct in all cases except when $p$ is true and $q$ is false. In regard of continuity, $p$ and $q$ are connected with a function $\mathrm{f}$, and it is essential to find suitable examples of functions illustrating the implication.

If the inequality $\left|x-x_{0}\right|<\delta$ is denoted with $p$, and the inequality $\left|f(x)-f\left(x_{0}\right)\right|<\varepsilon$ is denoted with $q$, then, the visual explanation of the implication $\left|x-x_{0}\right|<\delta \Rightarrow\left|f(x)-f\left(x_{0}\right)\right|<\varepsilon$ is:

The implication $\left|x-x_{0}\right|<\delta \Rightarrow\left|f(x)-f\left(x_{0}\right)\right|<\varepsilon$ is false only if the antecedent is true and the consequence is false. It means that the ordered pair $(x, f(x))$ which do not satisfy this implication, visually correspond to the points which belong to the vertical area $\mathrm{V}$, but do not belong to the horizontal area H. In Figure 3, these points belong to the parts of the plain denoted with NO. In all other parts of the plain (denoted with YES) there are points whose coordinates make the implication correct.

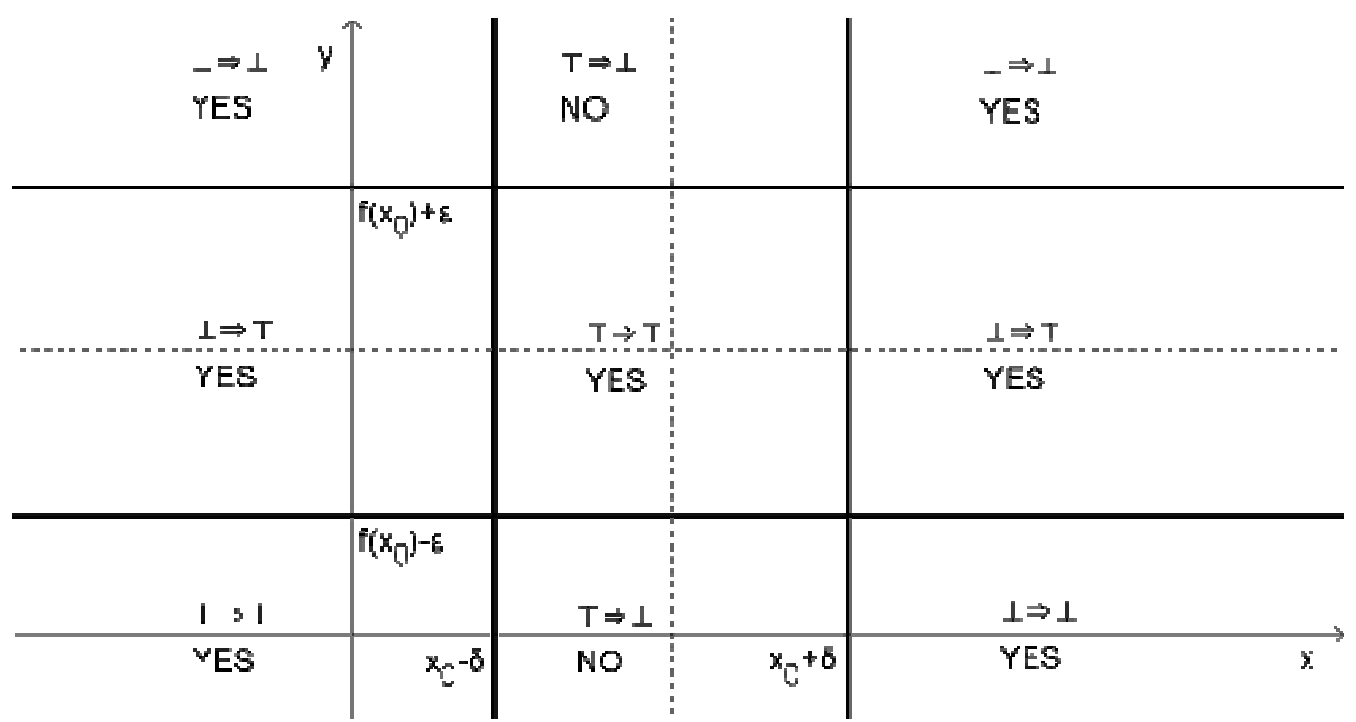

Figure 3. Visual presentation of the truth value of implication $\left|x-x_{0}\right|<\delta \Rightarrow\left|f(x)-f\left(x_{0}\right)\right|<\varepsilon$

\section{Project}

In order to ensure acceptance of the notion of the continuity and provide understanding about importance of rigor and precision in the definition, we have designed specific collaborative project.

Project was situated in mathematical high school and involved 21 students (students talented for mathematics). These students learn continuity in the second semester of the third grade, when they are approximately 18 years old.

In this project, seven formulae, given below, were analyzed with students, visualized and compared. Students were divided into seven groups and in each group there were three participants. Each group analyzed one of seven following formulae:

$$
\text { (1) }(\forall \varepsilon>0)(\exists \delta>0)\left(\forall x \in D_{f}\right)\left(\left|f(x)-f\left(x_{0}\right)\right|<\varepsilon \Rightarrow\left|x-x_{0}\right|<\delta\right) \text {, }
$$


(2) $(\exists \delta>0)(\forall \varepsilon>0)\left(\forall x \in D_{f}\right)\left(\left|f(x)-f\left(x_{0}\right)\right|<\varepsilon \Rightarrow\left|x-x_{0}\right|<\delta\right)$,

(3) $(\forall \delta>0)(\exists \varepsilon>0)\left(\forall x \in D_{f}\right)\left(\left|f(x)-f\left(x_{0}\right)\right|<\varepsilon \Rightarrow\left|x-x_{0}\right|<\delta\right)$,

(4) $(\exists \varepsilon>0)(\forall \delta>0)\left(\forall x \in D_{f}\right)\left(\left|f(x)-f\left(x_{0}\right)\right|<\varepsilon \Rightarrow\left|x-x_{0}\right|<\delta\right)$,

(5) $(\forall \delta>0)(\exists \varepsilon>0)\left(\forall x \in D_{f}\right)\left(\left|x-x_{0}\right|<\delta \Rightarrow\left|f(x)-f\left(x_{0}\right)\right|<\varepsilon\right)$,

(6) $(\exists \varepsilon>0)(\forall \delta>0)\left(\forall x \in D_{f}\right)\left(\left|x-x_{0}\right|<\delta \Rightarrow\left|f(x)-f\left(x_{0}\right)\right|<\varepsilon\right)$,

(7) $(\exists \delta>0)(\forall \varepsilon>0)\left(\forall x \in D_{f}\right)\left(\left|x-x_{0}\right|<\delta \Rightarrow\left|f(x)-f\left(x_{0}\right)\right|<\varepsilon\right)$.

These formulae result from the standard definition, by changing the order of symbols ( $\varepsilon$ and $\delta$ ), quantifiers $\left(\forall\right.$ and $\exists$ ) and inequalities $\left(\left|x-x_{0}\right|<\delta\right.$ and $\left.\left|f(x)-f\left(x_{0}\right)\right|<\varepsilon\right)$.

For each of these cases, students have to find a function which is continuous at a point $x_{0}$ from its domain, but the formula is not correct, and the function which has a discontinuity at a point $x_{0}$ from its domain, although the observed formula holds.

Students used interactive application GeoGebra as supporting tool in their work. (http://www.geogebra.org)

\section{Formulae 1, 2, 3 and 4}

In this section we consider the formulae containing the following implication:

$$
\left|f(x)-f\left(x_{0}\right)\right|<\varepsilon \Rightarrow\left|x-x_{0}\right|<\delta
$$

If positive $\varepsilon$ and $\delta$ are given, the implication $\left|f(x)-f\left(x_{0}\right)\right|<\varepsilon \Rightarrow\left|x-x_{0}\right|<\delta$ is false if and only if the antecedent is true and the consequent is false. It means that the ordered pair $(x, f(x))$ which do not satisfy this implication, visually correspond to the points which belong to the horizontal area $H$, but do not belong to the vertical area V. In Figure 4, these points belong to the shaded parts of the plain. In all other (not shaded) parts of the plain there are points whose coordinates make the implication correct.

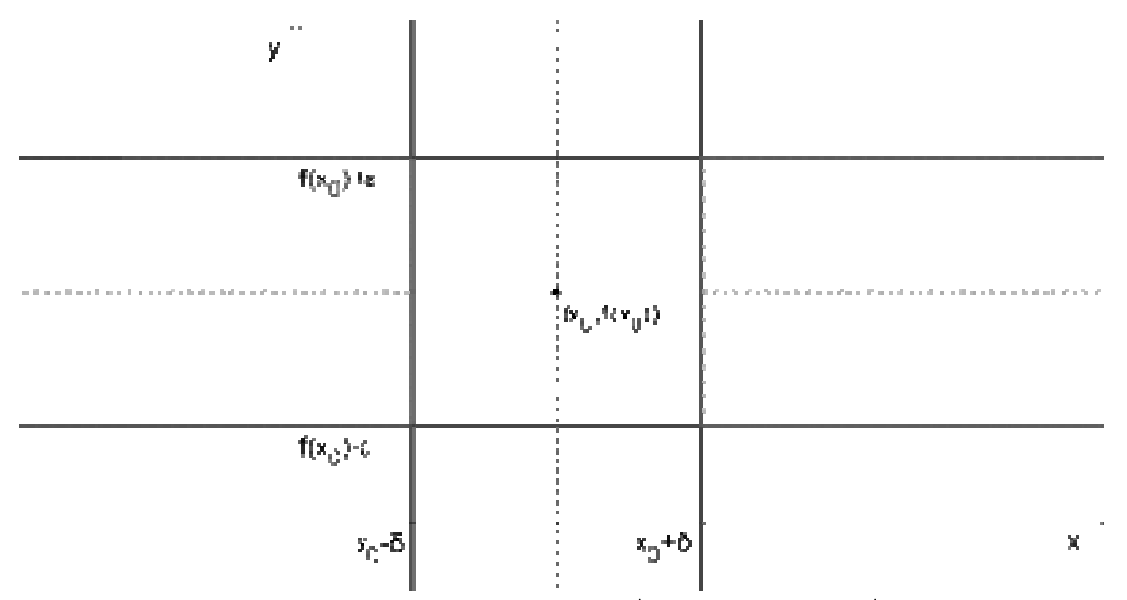

Figure 4. Visual presentation of implication $\left|f(x)-f\left(x_{0}\right)\right|<\varepsilon \Rightarrow\left|x-x_{0}\right|<\delta$

\section{Formulae 5, 6 and 7}

In these three formulae the order of implication is the same as in Definition 1:

$$
\left|x-x_{0}\right|<\delta \Rightarrow\left|f(x)-f\left(x_{0}\right)\right|<\varepsilon .
$$


The implication (3) means that the ordered pair $(x, f(x))$, which do not satisfy this implication, visually correspond to the points which belong to the vertical area $\mathrm{V}$, but do not belong to the horizontal area H. In Figure 5, these points belong to the shaded parts of the plain. In all other (not shaded) parts of the plain there are points whose coordinates make the implication correct.

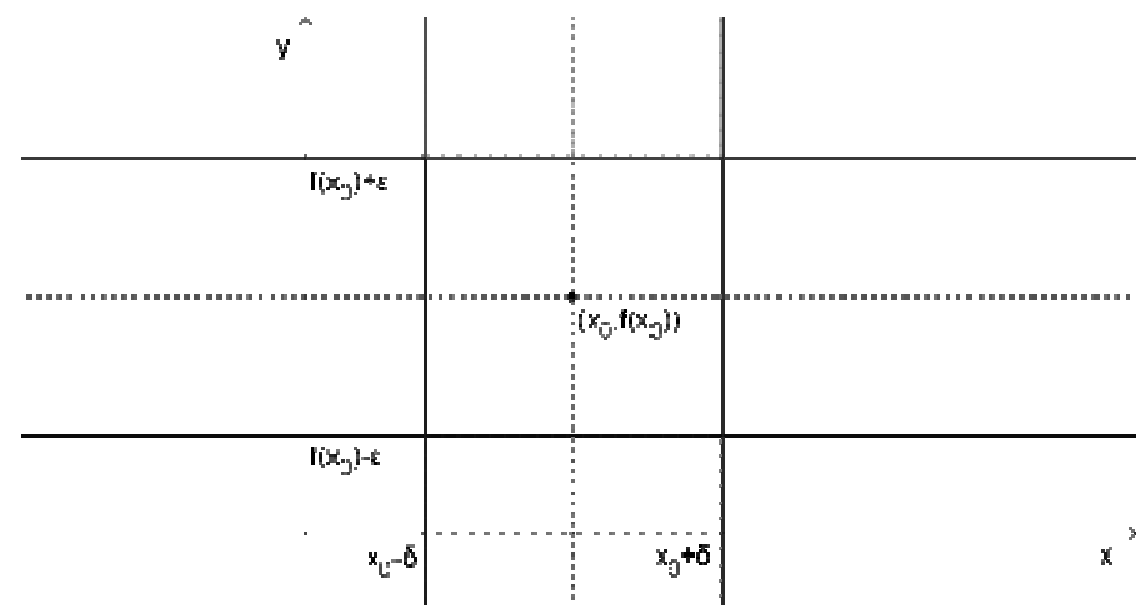

Figure 5. Visual presentation of implication $\left|x-x_{0}\right|<\delta \Rightarrow\left|f(x)-f\left(x_{0}\right)\right|<\varepsilon$

Next, the order of quantifiers and symbols is changed and each of these cases was analyzed.

\section{The results of the project}

In general, students were successful in solving specific tasks of the project. Their results are summarized in the following sections.

Detailed analysis of results is given for the Group 1, while in all other groups, only the examples of the functions are shown.

\section{The results of the Group 1 (Formula 1)}

In Formula 1, parameter $\delta$ depends on $\varepsilon$.

Let us compare the Formula 1: $(\forall \varepsilon>0)(\exists \delta>0)\left(\forall x \in D_{f}\right)\left(\left|f(x)-f\left(x_{0}\right)\right|<\varepsilon \Rightarrow\left|x-x_{0}\right|<\delta\right)$ with the formula from Definition 1 :

$$
(\forall \varepsilon>0)(\exists \delta>0)\left(\forall x \in D_{f}\right)\left(\left|x-x_{0}\right|<\delta \Rightarrow\left|f(x)-f\left(x_{0}\right)\right|<\varepsilon\right) .
$$

The difference between these two formulae is in the order of inequalities from the implication, but not in the order of quantifiers and symbols.

The visual meaning of the Formula 1 is:

For every height of the horizontal area $(\mathrm{H})$, there exists a vertical area $(\mathrm{V})$ of a certain width, such as the Figure of a function does not belongs to the part of the plain which is shaded in fig 4 (if the implication is true, then it is not permissible that part of the Figure belongs to the horizontal area $(\mathrm{H})$ but not belongs to the vertical area (V)).

In order to show that Formula 1 cannot be used as a definition of a continuous function at a point, it is given:

- the example of a discontinues function satisfying all conditions of the Formula 1;

- the example of a function which is continuous, but at some points, the Formula 1 is not correct.

Example 4.1 The function $f(x)=\left\{\begin{array}{cc}x, & x \leq 1 \\ x+1, & x>1\end{array}\right.$, has discontinuity at the point $x_{0}=1$, but it fulfils all conditions from Formula 1.

In the Figures 6(a) and 6(b) it is shown on two examples that, for every $\varepsilon$, we can choose $\delta:=\varepsilon$ such as the following proposition is true: 
If the point of the Figure of the function belongs to the horizontal area ( $\varepsilon$-high), then it belongs to the vertical one ( $\delta$-width).

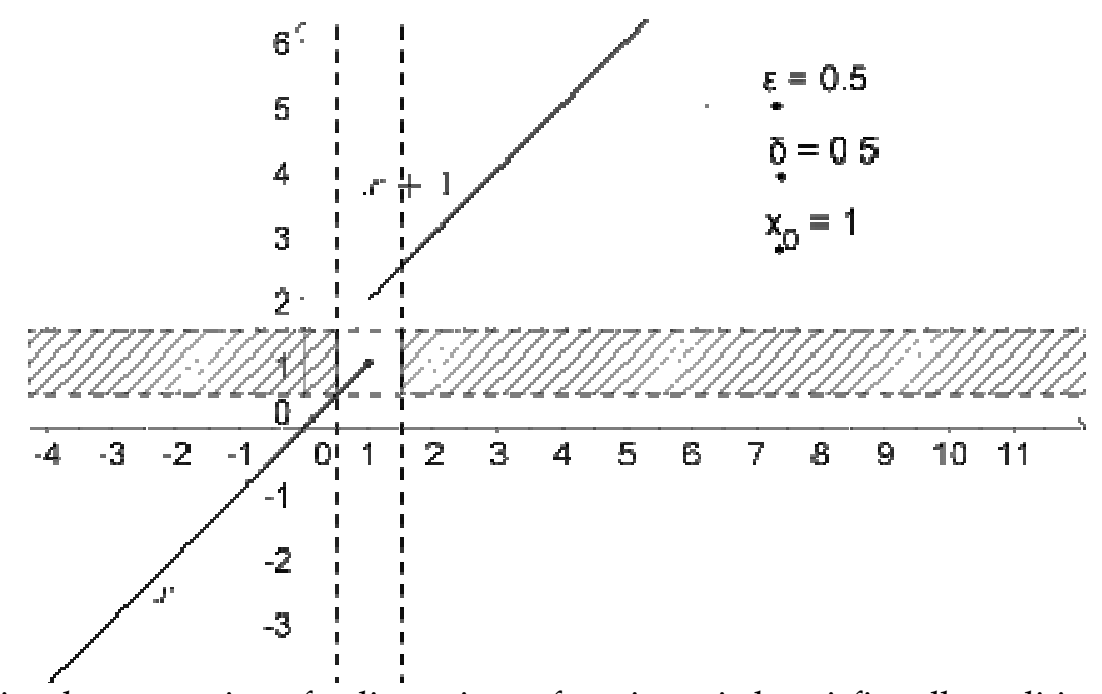

Figure 6(a). Visual presentation of a discontinues function witch satisfies all conditions of Formula 1 for $\varepsilon=0.5$ and $\delta=0.5$

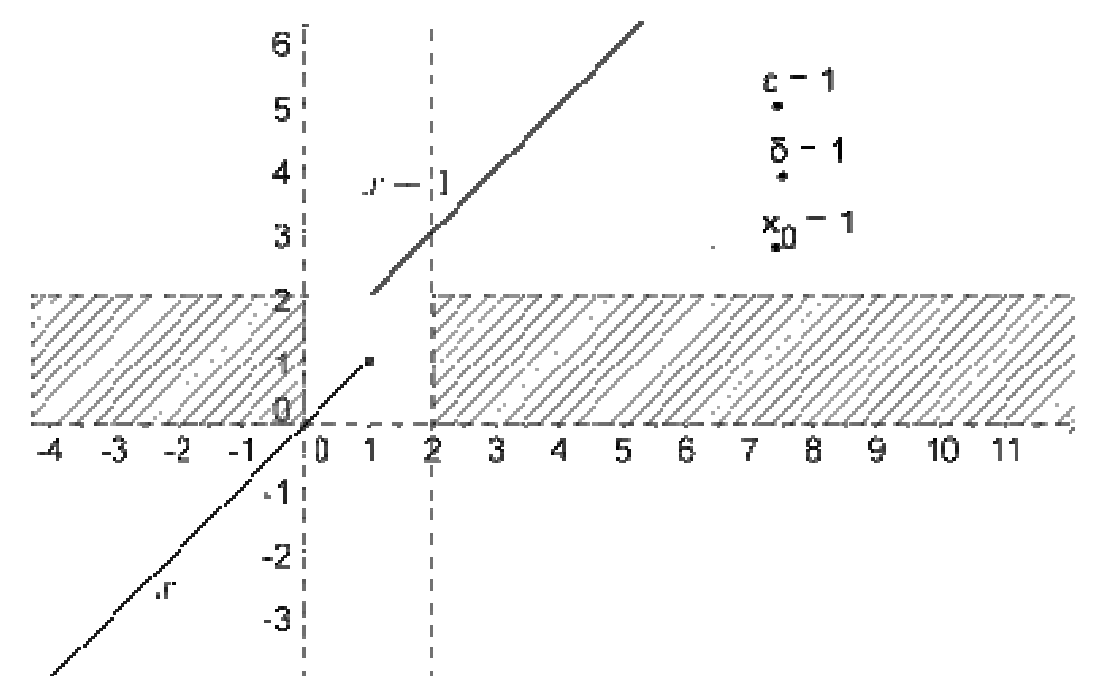

Figure 6(b). Visual presentation of a discontinues function witch satisfies all conditions of Formula 1 for $\varepsilon=1$ and $\delta=1$

Example 4.2 The function $f(x)=\frac{2}{3} \sin (x+0.25)+3$ is continuous at the point $x_{0}=3$, but at some points the formula is not correct. Its Figure is given in Figure 7. 


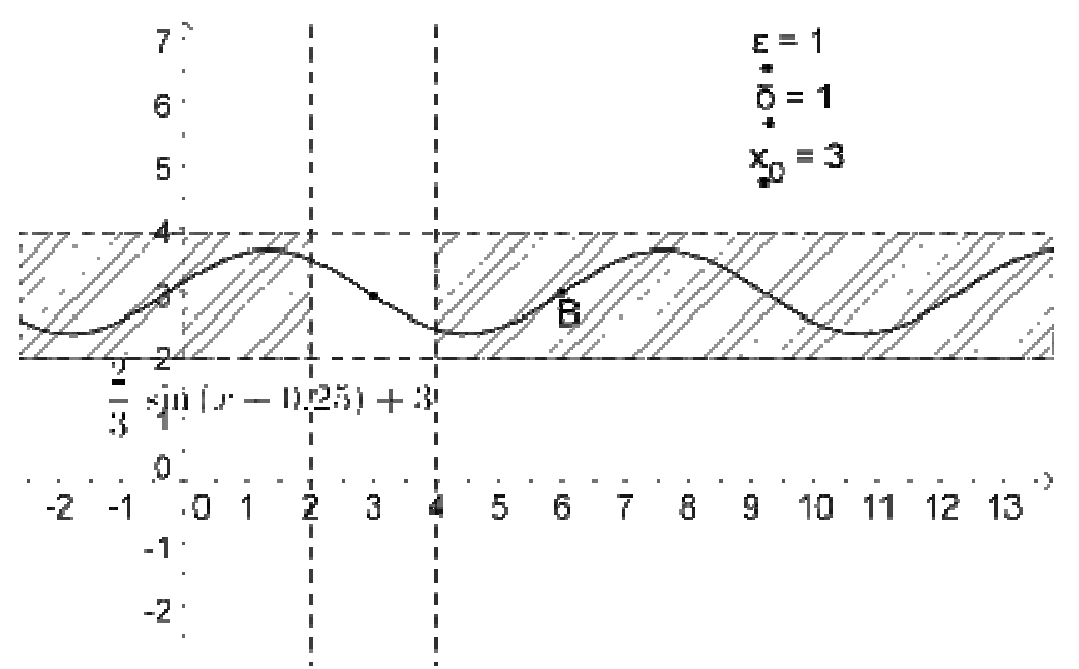

Figure 7. Visual presentation of a function which is continuous, but at some points the Formula 1 is not correct

We can see that for any change of width of the horizontal area $\mathrm{H}$ which contains the line $y=3$, there are always some points of the Figure of this function, for which Formula 1 is false. Namely, there are points of the Figure which belong to the horizontal, but not to the vertical area, for example from the fig. 8, the point $B=(6, f(6))$.

\section{The results of the Group 2 (Formula 2)}

If we compare Formula 2: $(\exists \delta>0)(\forall \varepsilon>0)\left(\forall x \in D_{f}\right)\left(\left|f(x)-f\left(x_{0}\right)\right|<\varepsilon \Rightarrow\left|x-x_{0}\right|<\delta\right)$ with the formula from Definition 1, then it is obvious that the difference between these two formulae is in the order of all elements: symbols, inequalities from the implication and quantifiers.

Example 4.3 The function $f:(-1,1) \rightarrow R, f(x)=\left\{\begin{array}{cc}\ln \frac{1+x}{1-x}, & x \in(-, 1,1) \backslash\{0\} \\ 1, & x=0\end{array}\right.$, has discontinuity at the point $x_{0}=0$, but it fulfils the conditions from Formula 2. (Figure 8)

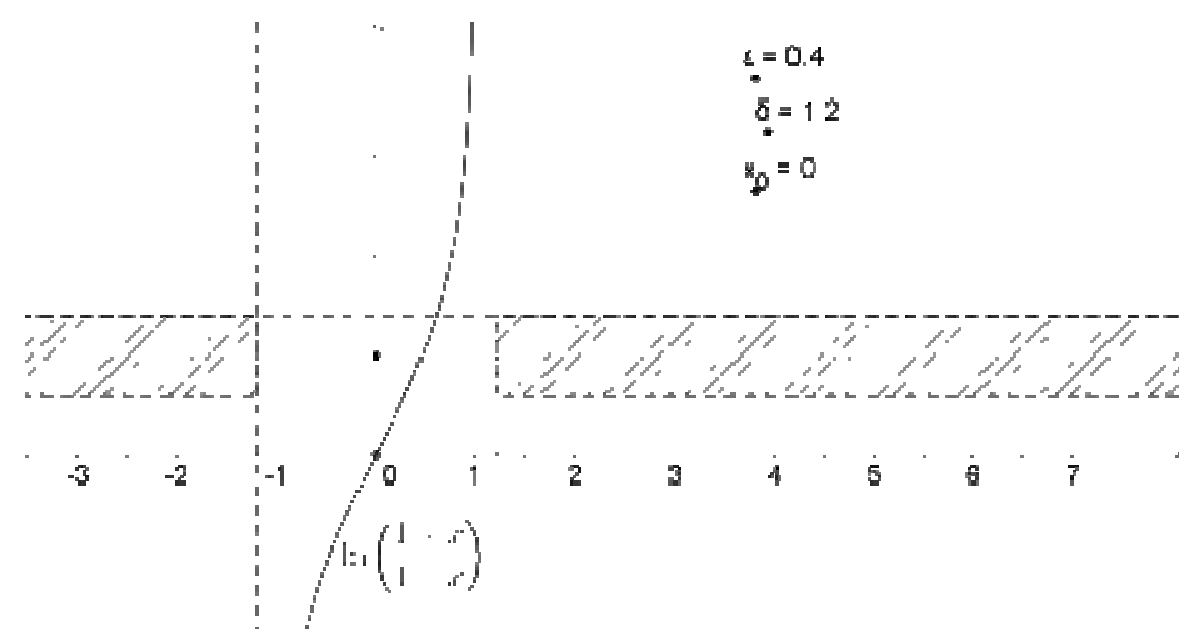

Figure 8. Visual presentation of a discontinues function witch satisfies all conditions of Formula 2

Example 4.4 The function $f(x)=x$ is continuous at the point $x_{0}=2$, but at some points from the Figure the formula 2 is not correct. (Figure 9) 


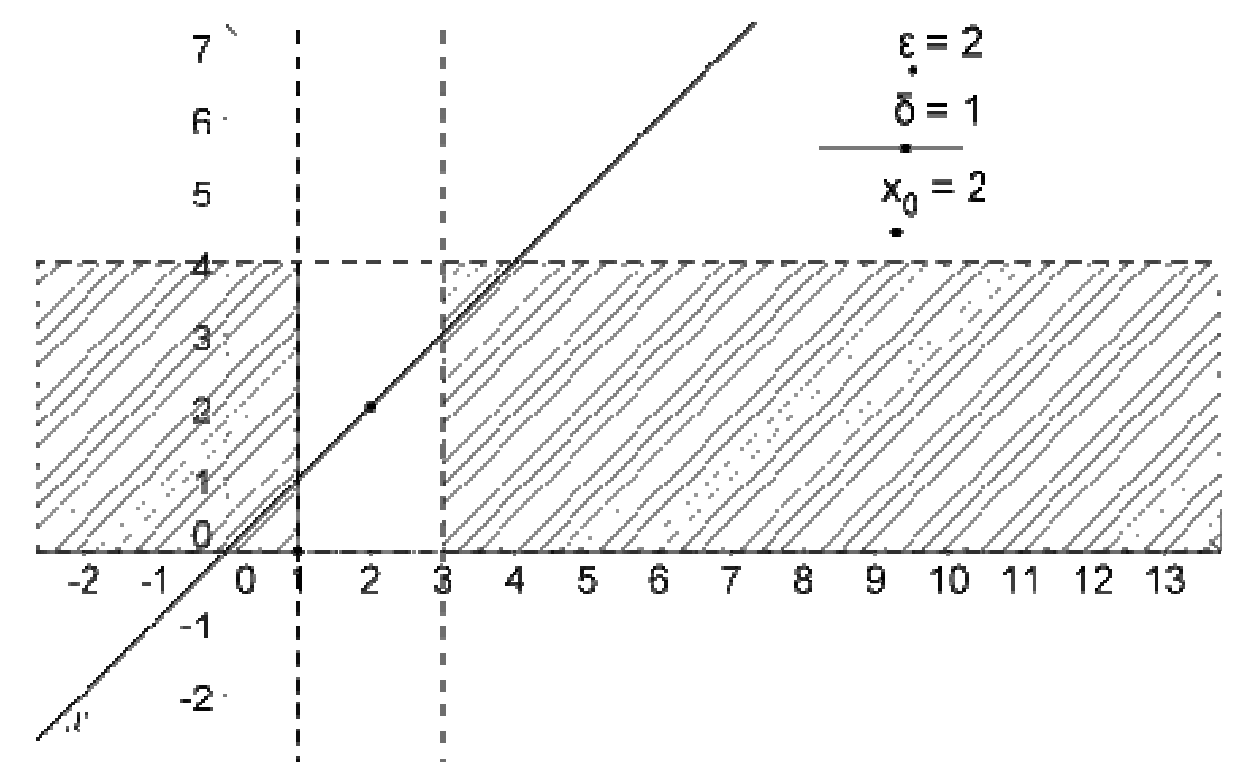

Figure 9. Visual presentation of a function which is continuous, but at some points the Formula 2 is not correct

The results of the Group 3 (Formula 3)

In Formula 3, the value of $\varepsilon$ depends on the value of $\delta$. If we compare Formula 3:

$$
(\forall \delta>0)(\exists \varepsilon>0)\left(\forall x \in D_{f}\right)\left(\left|f(x)-f\left(x_{0}\right)\right|<\varepsilon \Rightarrow\left|x-x_{0}\right|<\delta\right),
$$

with the formula from Definition 1, then we can see that the difference between these two formulae is in the order of symbols and inequalities from the implication.

Example 4.5 The function $f(x)=\left\{\begin{array}{ll}x^{2}, & x \neq 0 \\ -1, & x=0\end{array}\right.$, has discontinuity at the point $x_{0}=0$, but it fulfils the conditions from Formula 3. (Figure 10)

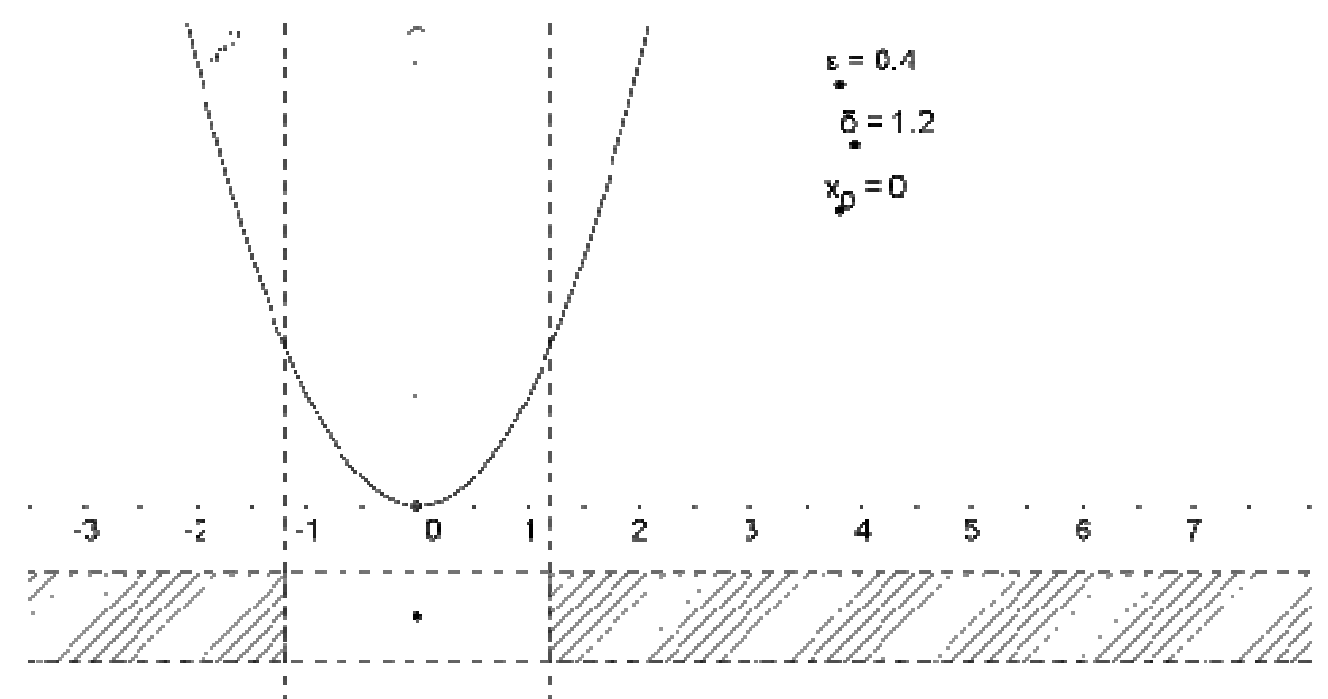

Figure 10. Visual presentation of a discontinues function witch satisfies all conditions of Formula 3

Example 4.6 The function $f(x)=2$ is continuous at the point $x_{0}=3$, but at some points from the Figure the formula 3 is not correct. (Figure 11) 


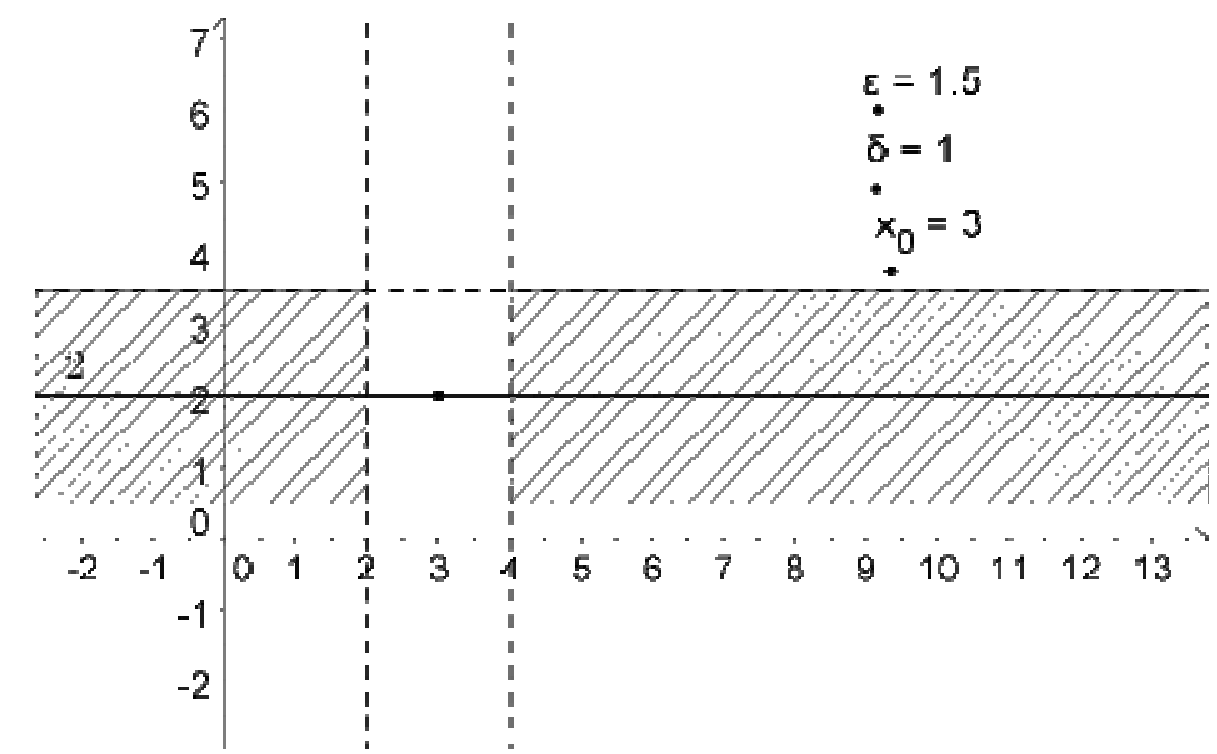

Figure 11. Visual presentation of a function which is continuous, but at some points the Formula 3 is not correct

The results of the Group 4 (Formula 4)

The difference between Formula 4:

$$
(\exists \varepsilon>0)(\forall \delta>0)\left(\forall x \in D_{f}\right)\left(\left|f(x)-f\left(x_{0}\right)\right|<\varepsilon \Rightarrow\left|x-x_{0}\right|<\delta\right),
$$

and formula from Definition 1, is in the order of quantifiers and inequalities from the implication. Formula 4 differs from Formula 3 only in the order of values $\varepsilon$ and $\delta$, and in this case $\varepsilon$ does not depend on $\delta$.

Example 4.7 The function $f(x)=\left\{\begin{array}{cl}x, & x<0 \\ 1.5, & x=0 \\ x+3, & x>0\end{array}\right.$, has discontinuity at the point $x_{0}=0$, but it fulfils the conditions from Formula 4. (Figure 12)

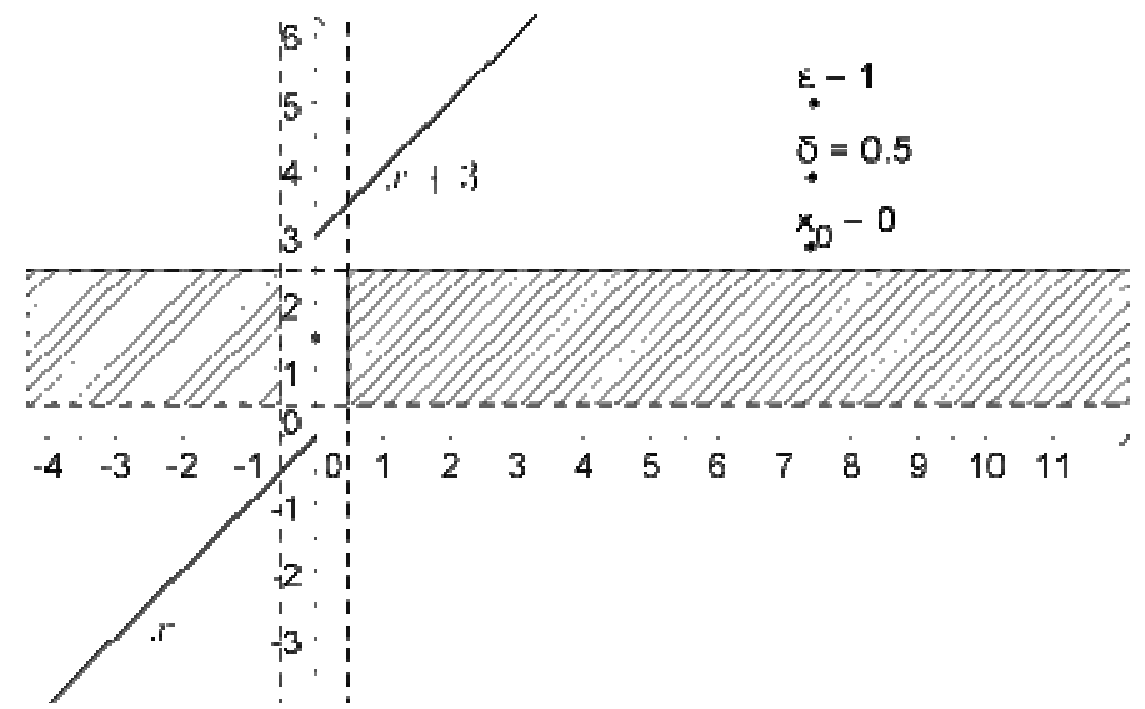

Figure 12. Visual presentation of a discontinues function which satisfies all conditions of Formula 4

Example 4.8 The function $f(x)=\sin x$ is continuous at the point $x_{0}=0$, but at some points from the Figure the formula 4 is not correct. (Figure 13) 


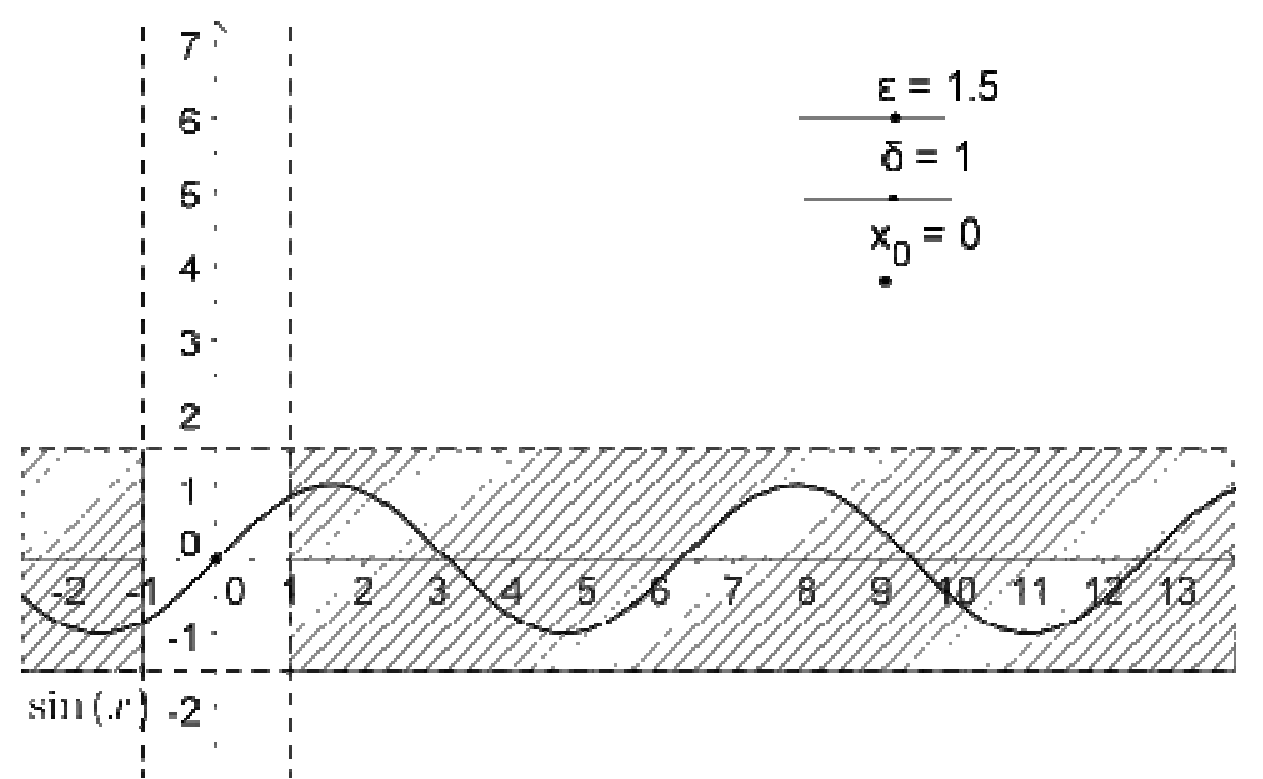

Figure 13. Visual presentation of a function which is continuous, but at some points the Formula 4 is not correct

The results of the Group 5 (Formula 5)

If we compare Formula 5:

$$
(\forall \delta>0)(\exists \varepsilon>0)\left(\forall x \in D_{f}\right)\left(\left|x-x_{0}\right|<\delta \Rightarrow\left|f(x)-f\left(x_{0}\right)\right|<\varepsilon\right),
$$

with the formula from Definition 1, then we can see that the difference between these two formulae is in the order of symbols ( $\varepsilon$ depends on $\delta$ ).

Example 4.9 The function $f(x)=\operatorname{sgn} x$, has discontinuity at the point $x_{0}=0$, but it fulfils the conditions from Formula 5. (Figure 14)

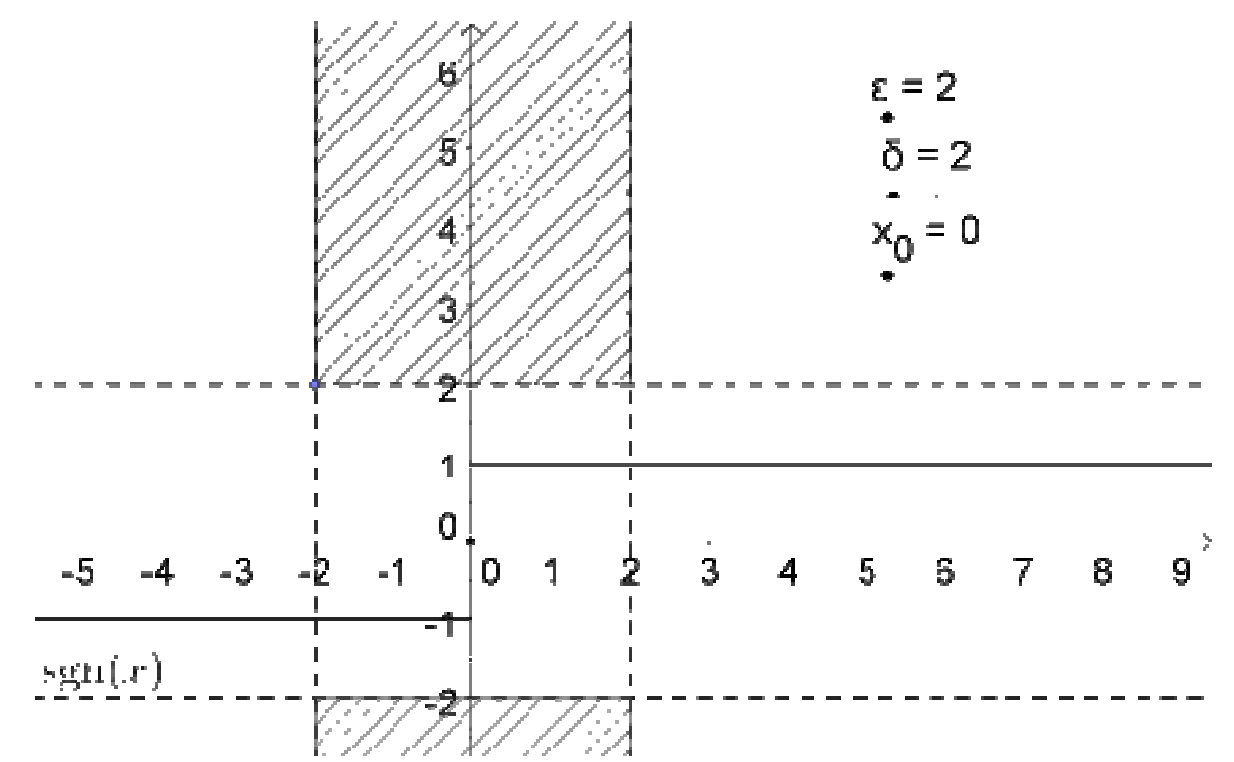

Figure 14. Visual presentation of a discontinues function which satisfies all conditions of Formula 5

Example 4.10 The function $f(x)=\tan x, x \neq \frac{k \pi}{2}, k \in Z$ is continuous at the point $x_{0}=0$, but at 
some points from the Figure the formula 5 is not correct. (Figure 15)

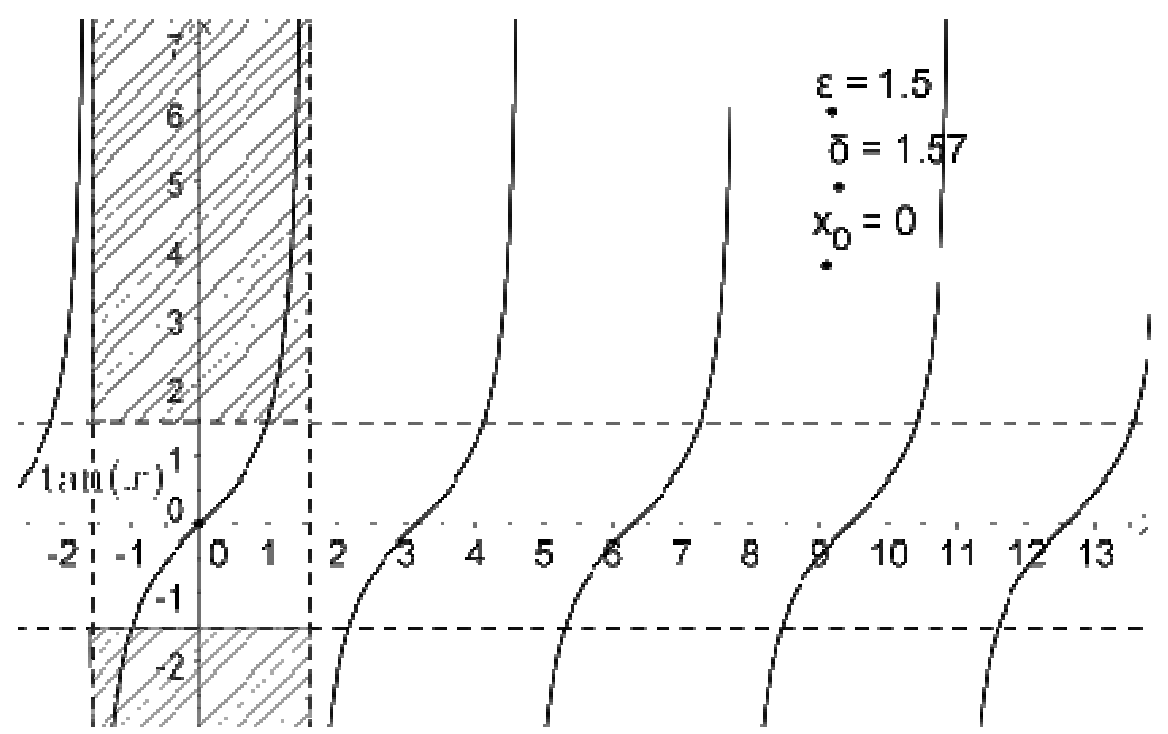

Figure 15. Visual presentation of a function which is continuous, but at some points the Formula 5 is not correct

The results of the Group 6 (Formula 6)

The difference between Formula 6:

$$
(\exists \varepsilon>0)(\forall \delta>0)\left(\forall x \in D_{f}\right)\left(\left|x-x_{0}\right|<\delta \Rightarrow\left|f(x)-f\left(x_{0}\right)\right|<\varepsilon\right),
$$

And the formula from Definition 1 is in the order of quantifiers and inequality. In Formula $6 \varepsilon$ do not depend on $\delta$.

Example 4.11 The function $f(x)=\left\{\begin{array}{ll}\sin x+2, & x \leq 0 \\ \cos x+2, & x>0\end{array}\right.$, has discontinuity at the point $x_{0}=0$, but it fulfils the conditions from Formula 6. (Figure 16)

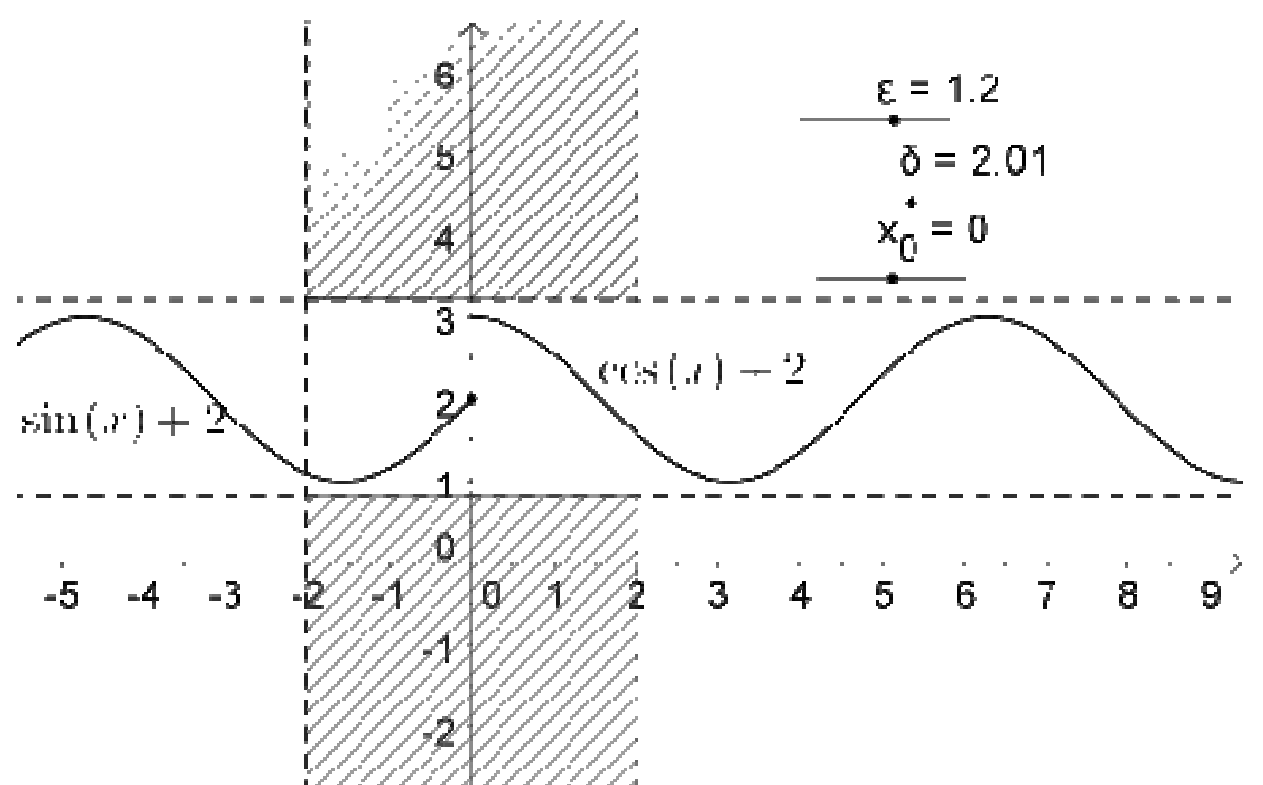

Figure 16. Visual presentation of a discontinues function which satisfies all conditions of Formula 6

Example 4.12 The function $f(x)=x^{3}$ is continuous at the point $x_{0}=0$, but at some points from the Figure the formula 6 is not correct. (Figure 17) 


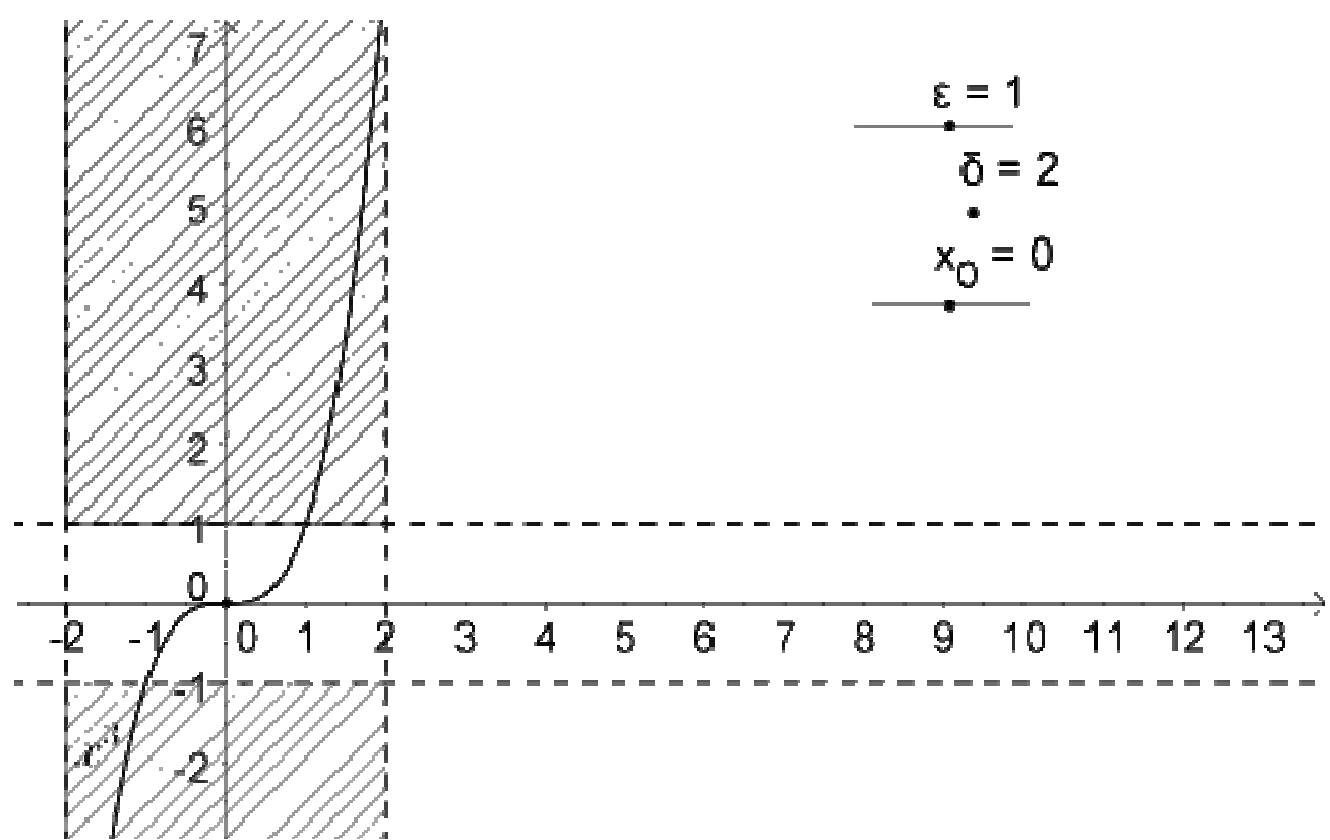

Figure 17. Visual presentation of a function which is continuous, but at some points the Formula 6 is not correct

The results of the Group 6 (Formula 6)

Finally, let us consider the Formula 7:

$$
(\exists \delta>0)(\forall \varepsilon>0)\left(\forall x \in D_{f}\right)\left(\left|x-x_{0}\right|<\delta \Rightarrow\left|f(x)-f\left(x_{0}\right)\right|<\varepsilon\right) .
$$

It differs from Definition 1 only in the order of quantifiers and symbols. The value of $\delta$ in Formula 7 do not depend on $\varepsilon$.

Example 4.13 The function $f(x)=2 x-2$ is continuous at the point $x_{0}=0$, but its Figure still contains some points for which coordinates Formula 7 is not true (e.g., for $\delta=1$ and $\varepsilon=1$ this formula is false). (Figure 18)

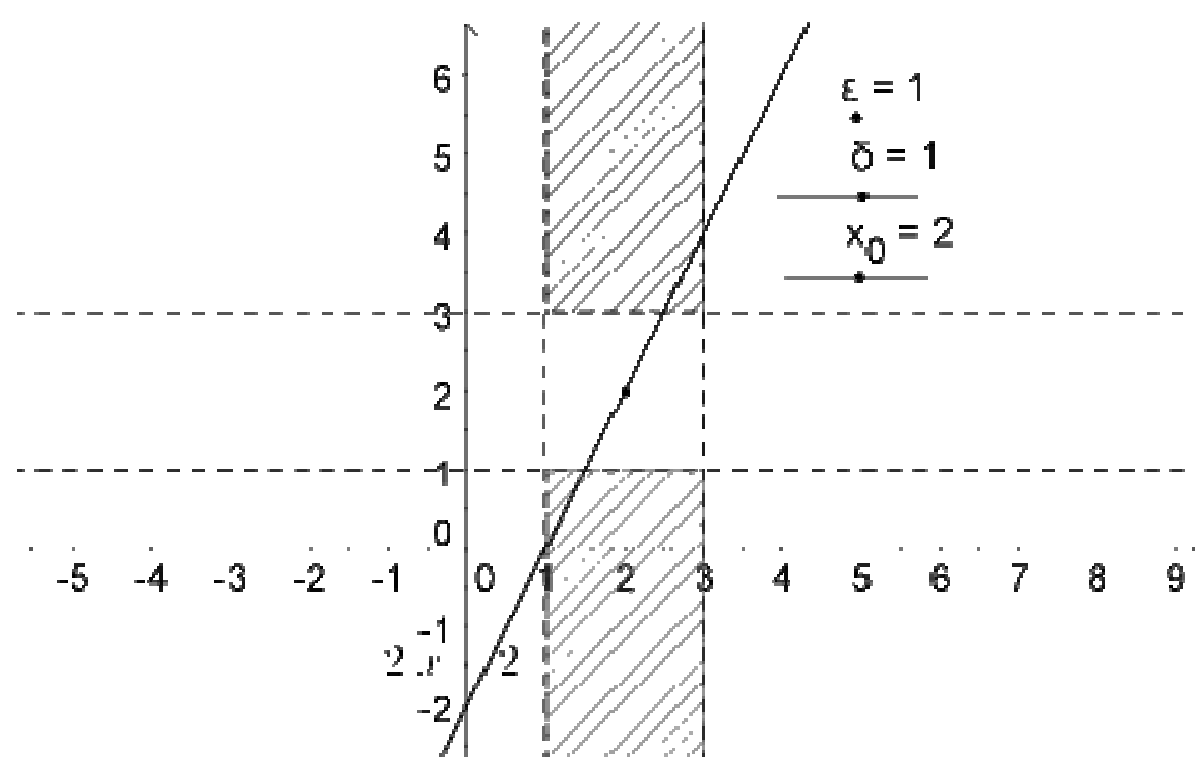

Figure 18. Visual presentation of a function which is continuous, but at some points the Formula 7 is not correct

As we expected, finding the example of a function that have discontinuity at some point and satisfies 
all conditions of Formula 7 was difficult task. However, students from the group 7 realized that it is necessary to consider a function with contains an isolated point. That conclusion further initiated a sensible discussion about continuity of a function at an isolated point.

\section{Conclusions}

In this paper we introduced and explained a visual approach in teaching and learning the epsilondelta definition of continuity which is focused on the reinforcement of mathematical skills and deepening students understanding of concept of continuity.

Although this approach is used as means to develop specific mathematical knowledge of students in mathematical high schools by building on student's prior understanding, it is also aimed to the cultivation of mathematical ingenuity and creativity by gradual and systematic guiding through the complexity of this demanding concept.

In that sense, we focus on efforts to create collaborative and student-centred environments where the teacher and the student are considered as partners in the teaching-learning process.

Due to the fact that collaborative visual teaching of epsilon delta definition is multi-faceted, the students think of continuity of the function more deeply and more broadly. Discussing explicitly each meaning of the elements from definition enables that students develop sophisticated understanding about this concept.

To summarize, we would like to emphasize the main advantages of proposed approach:

(1) Promoting visual thinking in the classroom,

(2) Providing both challenge and support by establishing cooperative procedures,

(3) Developing mathematically sophisticated ideas in mathematical inquiry about epsilon delta definition of continuity.

\section{Acknowledgements}

The authors are grateful to the students who participated in the project.

\section{References}

Cobb P., Yackel E., Wood T. A., (1992). Constructivist alternative to the representational view of mind in mathematics education. Journal for Research in Mathematics Education, 23, 12-33.

Gray E., Tall D., (2007). Abstraction as a natural process of mental compression. Mathematics Education Research Journal, 19 (2), 23-40.

Takači Đ., Pešić D., Tatar J., (2006). On the continuity of functions, International Journal of Mathematical Education in Science and Technology, Vol 37, No 7, 783-791.

Takači Đ., Pešić D., Tatar J., (2003). An Introduction to the continuity of functions using Scientific Workplace, The Teaching of Mathematic, Vol VI, 2, 105-112.

Tall D., Vinner A., (1981). Concept Image and Concept Definition in Mathematics with special reference to Limits and Continuity. Education Studies in Mathematics, 12 (2), 151-169.

Tall D., (1991). The Transition to Advanced Mathematical Thinking: Functions, Limits, Infinity, and Proof. In Grouws D.A. (ed.) Handbook of Research on Mathematics Teaching and Learning, (pp 495-511). New York: Macmillan. 